\title{
Two-site CCD observations and spectroscopy of HD 207331: a new $\delta$ Scuti variable in Cygnus
}

\author{
L. Fox Machado ${ }^{\text {a,* }}$, W.J. Schuster ${ }^{\mathrm{a}}$, C. Zurita ${ }^{\mathrm{b}}$, J. S. Silva ${ }^{\mathrm{a}}$, R. Michel ${ }^{\mathrm{a}}$ \\ ${ }^{a}$ Observatorio Astronómico Nacional, Instituto de Astronomía - Universidad Nacional Autónoma de México, Ap. P. 877, Ensenada, BC 22860, Mexico \\ ${ }^{b}$ Instituto de Astrofísica de Canarias C/Vía Láctea s/n, 38205 La Laguna, Tenerife, Spain
}

\begin{abstract}
The results of an observational campaign on the new $\delta$ Scuti pulsator HD 207331 are reported. The star was observed photometrically from August 26 to September 2, 2009 from the Observatorio San Pedro Mártir (0.84-m telescope, Mexico) and the Observatorio del Teide (0.80-m telescope, Spain). An overall run of $53.8 \mathrm{~h}$ of useful data was collected from the two sites. Four oscillation frequencies for HD 207331 have been found above a 99\% confidence level. These results confirm the multiperiodic pulsation nature of the star suggested in previous observations with sparse data. Spectroscopic observations carried out in 2009 allowed us to derive its spectral type and luminosity class as well as to estimate its rotation rate. A simple comparison with models is performed.
\end{abstract}

Keywords: stars: $\delta$ Sct - techniques: photometric, spectroscopic - stars:oscillations - stars: individual: HD 207331, BD+42 4208, 7 Aql, 8 Aql.

'PACS: 97.30.Dg, 97.10.Ri, 97.10.Vm, 97.10.Zr, 97.10.Sj

\section{Introduction}

The $\delta$ Scuti-type pulsators are stars with masses between 1.5 and $2.5 M_{\odot}$ located at the intersection of the classical Cepheid instability strip with the main sequence. They have spectral types $\mathrm{A}$ and $\mathrm{F}$, a period range between $0.5 \mathrm{~h}$ and $6 \mathrm{~h}$, and generally pulsate with a large number of radial and nonradial modes excited by the $\kappa$-mechanism associated with the second Helium ionization zone. These oscillation modes penetrate to different depths inside the star. Thus, $\delta$ Scuti stars provide a good opportunity to probe the internal structure of intermediate mass stars.

Since most of the $\delta$ Scuti stars are short period variables with typical photometric amplitude of $20 \mathrm{mmag}$, their oscillations can be easily detected from the ground. In fact, several $\delta$ Scuti 'stars have been discovered accidentally when taken as reference stars for observations of well known $\delta$ Scuti stars [e.g. Fox Machado et al. (2002, 2007); Li et al. (2002)]. However to resolve their rich oscillation spectrum from the ground, high quality long time series are required which can only be obtained by means of observations from different sites distributed in longitude around the Earth (e.g. Li et al. 2004; Costa et al. 2007). Therefore, to characterize the pulsation spectrum of a new $\delta$ Scuti star additional observational efforts are needed.

In this paper we present the results of photometric and spectroscopic follow-up observations of HD 207331, a new $\delta$ Scuti star recently discovered in Cygnus, aimed at characterizing its pulsation behavior more accurately.

\footnotetext{
${ }^{*}$ Corresponding author. Tel.: +52 6461744580; fax +52 6461744607

Email address: 1 fox@astrosen. unam.mx (L. Fox Machado)
}

\section{The object HD 207331}

The star HD 207331 (= SAO 51294, BD+42 4207, HIP 107557) was discovered to be a new $\delta$ Scuti star by Schuster et al. (2008) when observing a sample of A-type stars on the night of September 27, 2007, in order to test the sixchannel $u v b y-\beta$ spectrophotometer attached to the H. L. Johnson 1.5-m telescope of the San Pedro Mártir Observatory, Baja California, Mexico. The variability of the star was clear, despite the fact that it was observed less than $2 \mathrm{~h}$. Six hours of CCD photometric observations on the night of September 30, 2007, with the $0.84-\mathrm{m}$ telescope of the same observatory confirmed its variability (Schuster et al., 2008).

Differential and standard Strömgren $(u v b y)$ photometry of HD 207331 was obtained by Fox Machado et al. (2008) using the same equipment as the former observations. In particular, the star was monitored for about $11 \mathrm{~h}$ between November 11 and November 19, 2007. As a result, Fox Machado et al. (2008) found evidence of at least two close frequencies which might explain the resulting beating behavior of the light curve. The derived standard photometry is the following: ( $V, b-y, m_{1}$, $\left.c_{1}\right)=(8.329,0.125,0.150,1.018)$. Fox Machado et al. (2008) also estimated a stellar reddening from the reddening maps of Schlegel et al. (1998) via the web-page calculator of the NED (NASA/IPAC Extragalactic Database). The derived intrinsic colours of HD 207331 are the followings $\left(V_{0},(b-y)_{0}, m_{0}, c_{0}\right)$ $=(7.980,0.044,0.174,1.002)$, and $\beta=2.854$, which yield a $M_{V}=1.058 \mathrm{mag}$, and $d=242 \mathrm{pc}$.

The Hipparcos catalogue (Perryman, et al., 1997), on the other hand, provides a parallax of $3.31 \pm 0.88$ mas, from which a distance value of $302 \pm 81$ pc can be estimated. This corre- 


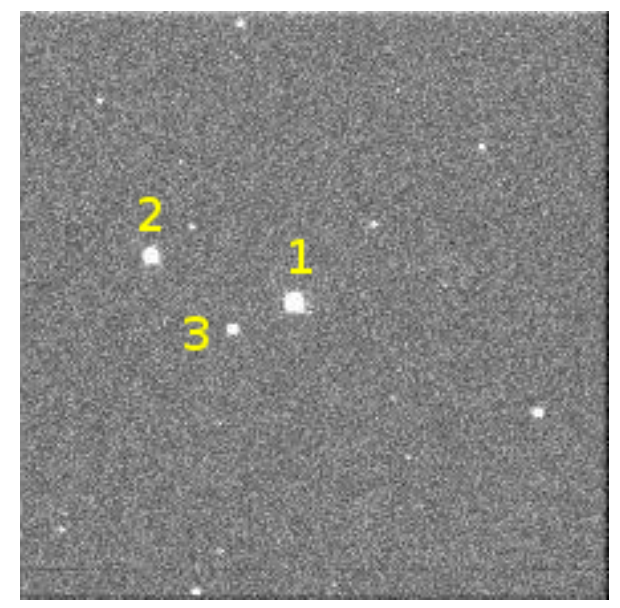

Figure 1: CCD imagen of the HD $207331 \mathrm{FOV}$ at the $0.84 \mathrm{~m}$ telescope. 1 stands for HD 207331, 2 for the comparison star, and 3 for the check star. Some properties of the stars are listed in Table 1. North is up and East is left.

sponds to a distance modulus of $7.40 \pm 0.60 \mathrm{mag}$, which indicates a value of $M_{v}=1.0 \pm 0.6 \mathrm{mag}$ for HD 207331. Therefore the Hipparcos distance is in agreement within 1- $\sigma$ error with distance reported by Fox Machado et al. (2008). The Hipparcos catalogue also lists for HD 207331 a magnitude of $H_{p}$ of $8.3970 \pm 0.0022 \mathrm{mag}$ (median error), $\pm 0.019 \mathrm{mag}$ (scatter) and a $V_{T}$ of $8.335 \pm 0.009$ mag (standard error).

Concerning the spectral classification of HD 207331, the SAO Star Catalog J2000 (SAO Staff 1966; USNO, ADC 1990) lists A0.

\section{Observations and data reduction}

\subsection{CCD photometric observations}

The two observatories involved in the observational campaign are the following:

-Observatorio del Teide (OT, Spain): the data were collected using the IAC 80 telescope $(0.80-\mathrm{m})$. The images were acquired with CAMELOT, a CCD detector of $2048 \times 2048$ pixels. Observer: CZ.

-Observatorio de San Pedro Mártir (SPM, Mexico): the observations were performed using the $0.84-\mathrm{m}$ telescope. The CCD was a SITe 1 of $1024 \times 1024$ pixels. Observers: WJS, JS, LFM. Observer's abbreviations correspond to the initials of the co-authors.

Table 1 gives the log of observations. Bad weather conditions at the OT did not allow us to get more than one night of data. However, a total amount of $53.8 \mathrm{~h}$ of useful data was obtained from the two sites.

The observations were obtained through a Johnson $B$ filter. Figure 1 shows a typical image of the CCD's field of view $\left(6.8 .^{\prime} \times 6.8^{\prime}\right)$ at the $84-\mathrm{cm}$ telescope of the San Pedro Mártir observatory. The target star is labeled with number 1 ; comparison and check stars with 2 and 3, respectively. As can be seen this is an uncrowded star field. Although the CCD's

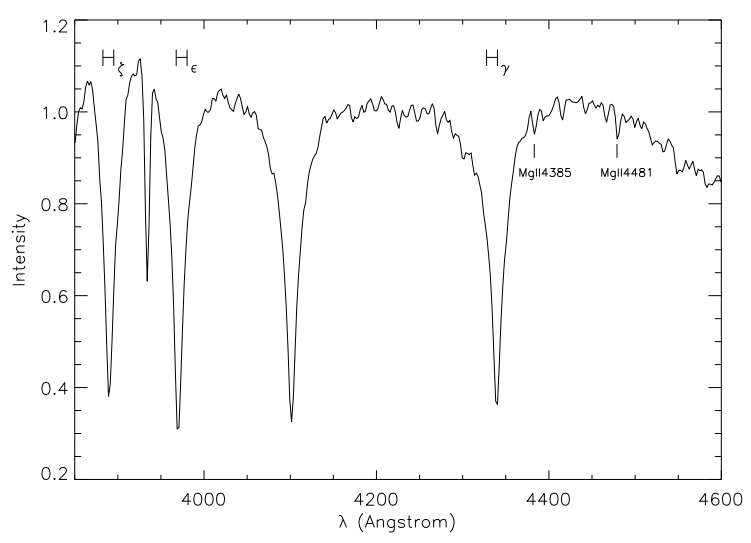

Figure 3: Normalized spectrum of HD 207331. For sake of clarity only the region between $3800 \AA-4600 \AA$ is shown.

FOV at the IAC-80 telesope of the Teide observatory is larger $\left(\sim 11^{\prime} \times 11^{\prime}\right)$, no other suitable comparison stars could be observed. Table 2 shows the main observational parameters corresponding to the target and comparison stars as taken from the SIMBAD database operated by the CDS (Centre de Données astronomique de Strasbourg).

Sky flats, dark and bias exposures were taken every night at both sites. All data were calibrated and reduced using standard IRAF routines. Aperture photometry was implemented to extract the instrumental magnitudes of the stars. The differential magnitudes were normalized by subtracting the mean of differential magnitudes for each night. In Figure 2 the entire light curves, HD 207331 - Comparison, are presented. As can be seen from the fourth panel (from top to bottom) no overlapping of the observations was obtained for the one night of observing at OT, the 28th of August 2009.

\subsection{Spectroscopic observations}

Spectroscopic observations were conducted at the 2.12-m telescope of the SPM observatory during September 27, 2009 (UT). We used the Boller \& Chivens spectrograph installed in the Cassegrain focus of the telescope. The 600 lines/mm gratting was used to cover a wavelength range from 3900 to 6000 $\AA$. A dispersion of $2.05 \AA$ per pixels with a resolution of 5.0 $\AA$ was employed. The SITe3 $1024 \times 1024$ pixel CCD with a $0.24 \mu \mathrm{m}$ pixel size was attached to the spectrograph. The spectra were reduced in the standard way using the IRAF package. Fig. 3 shows a close-up of the normalized spectrum at region $3800 \AA-4600 \AA$. The spectral type was derived by comparing the normalized spectra with those of well classified stars available in the literature. An additional check of the spectral type was done from the ratio of lines $\mathrm{Mg}$ II 4481 and $\mathrm{Mg}$ II 4385. We have derived a spectral type of A0V for HD 207331.

The projected rotational velocity of the star $(v \sin i)$ can be estimated from the empirical calibration $v$ sin $i$ vs FWHM given by Bush \& Hint (2008) who determined the $v \sin i$ of $118 \delta$ Scuti variables. To do so, we have measured the FWHM for the 

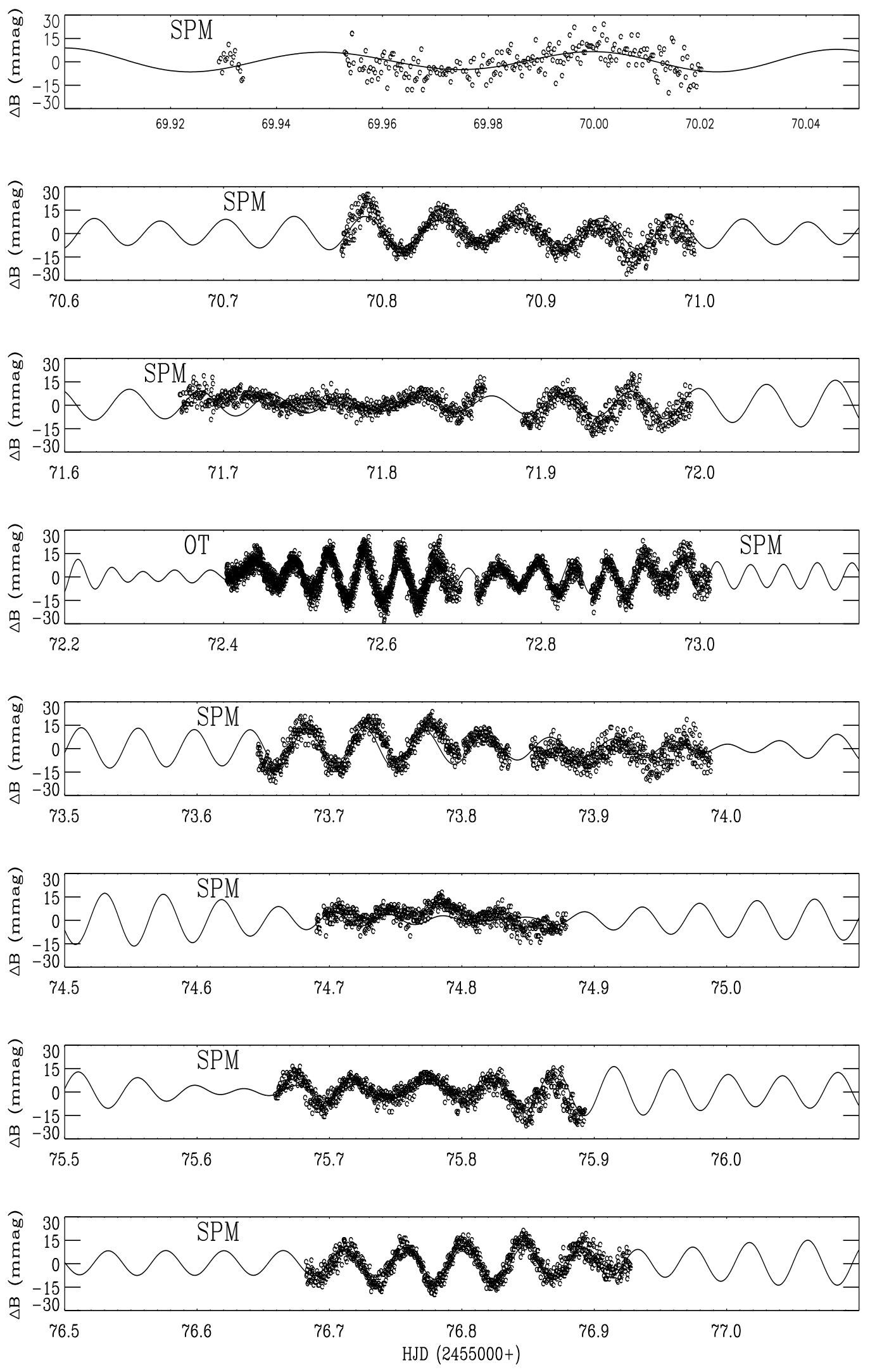

Figure 2: Differential light curves of HD 207331. The name of the observatory involved is indicated in each panel. 
Table 1: Log of observations. Observing time is expressed in hours.

\begin{tabular}{cccccc}
\hline Day & UT Date 2009 & $\begin{array}{c}\text { Start Time } \\
(\text { HJD 2455000+) }\end{array}$ & $\begin{array}{c}\text { End Time } \\
\text { (HJD 2455000+) }\end{array}$ & OT & SPM \\
\hline 1 & Aug 26 & 69.93 & 70.02 & - & 2.185 \\
2 & Aug 27 & 70.77 & 71.00 & - & 5.335 \\
2 & Aug 28 & 71.67 & 72.70 & 7.086 & 7.731 \\
4 & Aug 29 & 72.72 & 73.01 & - & 7.076 \\
5 & Aug 30 & 73.65 & 73.99 & - & 8.218 \\
6 & Aug 31 & 74.69 & 74.88 & - & 4.536 \\
7 & Sep 01 & 75.66 & 75.89 & - & 4.592 \\
8 & Sep 02 & 76.68 & 76.93 & - & 7.085 \\
\hline \multicolumn{7}{c}{} & Begin & End & Total Time & OT & SPM \\
& Aug 26 & Sep 02 & 53.844 & 7.086 & 46.758 \\
\hline
\end{tabular}

Table 2: Position, magnitude, and spectral type of target, comparison, and check stars observed in the CCD frame.

\begin{tabular}{lccccc}
\hline Star & ID & RA & Dec & V & SpTyp \\
& & $(2000.0)$ & $(2000.0)$ & $(\mathrm{mag})$ & \\
\hline Target & HD 207331 & 214702 & +431919 & 8.3 & $A 0$ \\
Comparison & BD+42 4208 & 214712 & +431951 & 9.4 & $A 0$ \\
Check & TYC 3196-1243-1 & 214706 & +431858 & 10.9 & - \\
\hline
\end{tabular}

$4501 \AA$ A Ti II and $4508 \AA$ Fe II lines. Then, we have applied the relationship (Bush \& Hintz, 2008):

$$
v \sin i=-33.7 F W H M^{2}+226.0 F W H M-215.3
$$

As a result a $v \sin i=133 \pm 10 \mathrm{~km} / \mathrm{s}$ was derived for HD 207331 .

In order to test the validity of the calibration for our telescope system we also have derived the $v \sin i$ values for $\delta$ Scuti variables $7 \mathrm{Aql}$ and $8 \mathrm{Aql}$ whose spectra were recorded by Fox Machado et al. (2010) with the same telescope, equipment and configuration as ours. These stars have accurately determined $v \sin i$ in the literature. We have found very good agreement between the derived projected rotational velocities from equation (1) and those listed in the literature for $7 \mathrm{Aql}$ and 8 Aql.

\section{Period analysis}

The period analysis has been performed by means of standard Fourier analysis and least-squares fitting. In particular, the amplitude spectra of the differential time series were obtained by means of Period04 package (Lenz \& Breger, 2005), which utilizes Fourier as well as multiple least-squares algorithms. This computer package allows us to fit all the frequencies simultaneously in the magnitude domain.

The spectral window in amplitude of the observations is shown in the first plot of Fig. 4. The amplitude spectrum of the differential light curve, HD 207331 - Comparison is depicted in the next plot. The subsequent plots in the figure, from left to right, illustrate the prewhitening process of the frequency peaks in each amplitude spectrum.

The frequencies have been extracted by means of a standard prewhitening method. In order to decide which of the detected
Table 3: Frequency peaks detected in the light curve: HD 207331 - Comparison. $\mathrm{S} / \mathrm{N}$ is the signal-to-noise ratio in amplitude after the prewhitening process.

\begin{tabular}{|c|c|c|c|c|c|c|}
\hline & $\begin{array}{l}\text { Freq. } \\
(\mathrm{c} / \mathrm{d})\end{array}$ & $\begin{array}{l}\text { Freq. } \\
(\mu \mathrm{Hz})\end{array}$ & $\begin{array}{c}\text { Period } \\
\text { hours }\end{array}$ & $\begin{array}{c}\mathrm{A} \\
(\mathrm{mmag})\end{array}$ & $\varphi /(2 \pi)$ & $S / N$ \\
\hline$f_{1}$ & 22.4880 & 260.186 & 0.937 & 7.76 & 0.18 & 13.7 \\
\hline$f_{2}$ & 20.0923 & 232.468 & 0.837 & 3.02 & 0.90 & 5.1 \\
\hline$f_{3}$ & 24.5384 & 283.909 & 1.022 & 3.22 & 0.32 & 6.3 \\
\hline$f_{4}$ & 23.7409 & 274.682 & 0.989 & 3.18 & 0.85 & 5.9 \\
\hline
\end{tabular}

peaks in the amplitude spectrum can be regarded as intrinsic to the star, Breger's criterion has been followed (Breger et al., 1993), where it was shown that the signal-to-noise ratio (in amplitude) should be at least 4 in order to ensure that the extracted frequency is significant.

The frequencies, amplitudes, and phases are listed in Table 3 . Four significant frequencies have been detected in HD 207331. A comparison of this four-frequency solution to the data is displayed in Fig. 2 with the solid line.

\section{Discussion}

From the differential light curves it can be noted that the beating behavior seen in the short time series of Fox Machado et al. (2008) is present. This is a common characteristic of the light curves of $\delta$ Scuti stars. The amplitude spectrum shows a concentration of high signal-to-noise peaks between 18-28 cycle day $^{-1}$. In particular, the highest amplitude peak is located at $22.49 \mathrm{~cd}^{-1}(260.19 \mu \mathrm{Hz})$, and the next significant frequency is located at $20.09 \mathrm{~cd}^{-1}(232.47 \mu \mathrm{Hz})$.

Fox Machado et al. (2008) detected a significant frequency peak at $\sim 21.1 \mathrm{~cd}^{-1}(244.1 \mu \mathrm{Hz})$ with an amplitude of $6 \mathrm{mmag}$ in Strömgren $y$ band. As can be seen in Table 3 this is well 

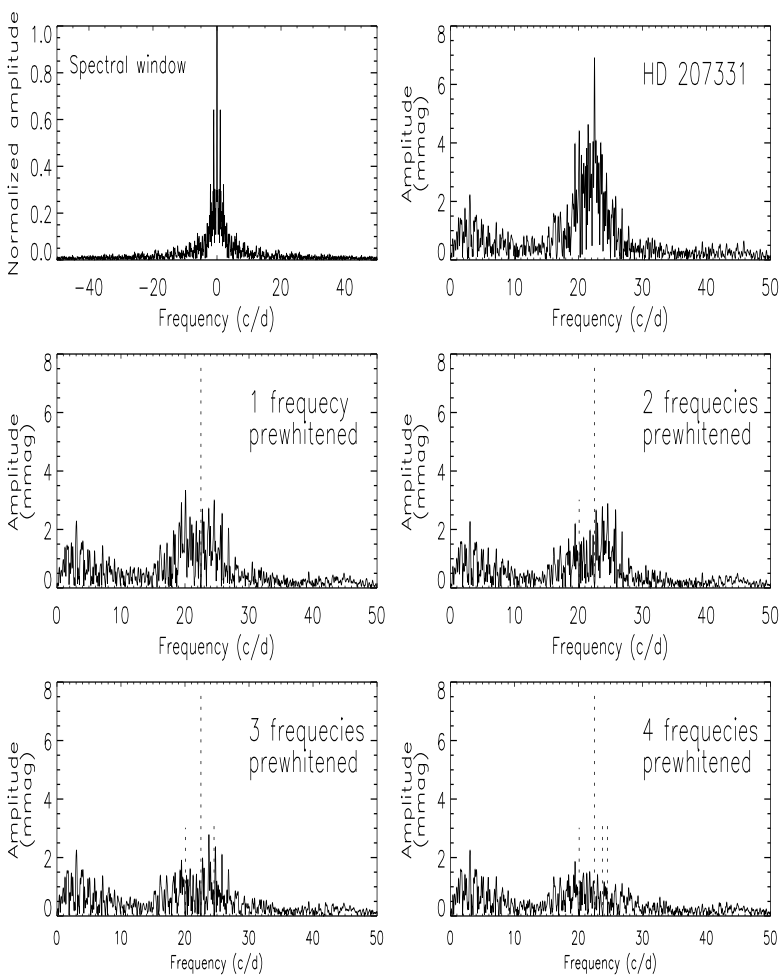

Figure 4: Spectral window (first plot). Pre-whitening process in HD 207331 (from second plot). In each plot, from left to right, the highest amplitude peak is selected and removed from the time series, and a new spectrum is obtained.

within the period of the modes detected in this campaign, taking into account that the complex window function of those observations does not allow a precise comparison. Nonetheless, this peak is most likely a side-lobe of the second frequency $\left(f_{2}\right)$ detected in our campaign. No equally spaced close frequency pairs were found in the frequency pattern of HD 207331.

\subsection{Preliminary comparison with theoretical models}

Figure 5 shows the observed position of HD 207331 (asterisk) in the HR diagram and its associated uncertainty (cross upon the asterisk). Since the Hipparcos distance for HD 207331 has a large relative error, we have considered in Fig. 5] the Stromgren absolute magnitude derived by Fox Machado et al. (2008), namely $M_{V}=1.058$. Error bars of $0.1 \mathrm{mag}$ for $M_{V}$ and $0.02 \mathrm{mag}$ for $(B-V)$ have been adopted.

Two main sequence evolutionary tracks that approximately match the observed position of HD 207331 are shown by dashed and dot-dashed lines. These evolutionary sequences were computed as explained in Fox Machado et al. (2006) by using the CESAM evolution code (Morel, 1997) with input physics appropriate to $\delta$ Scuti stars and a chemical initial composition of $Z=0.02$ and $Y=0.28$. Also shown are the observed instability strip boundaries from Rodríguez et al. (1994). According to the models depicted in Fig. 5, for a solar metallicity, HD 207331 would have a mass between $2.20 M_{\odot}$ and 2.25 $M_{\odot}$. The age of HD 207331 corresponding to these evolutionary models is $\sim 527 \mathrm{Myr}$ and $\sim 513 \mathrm{Myr}$, respectively.

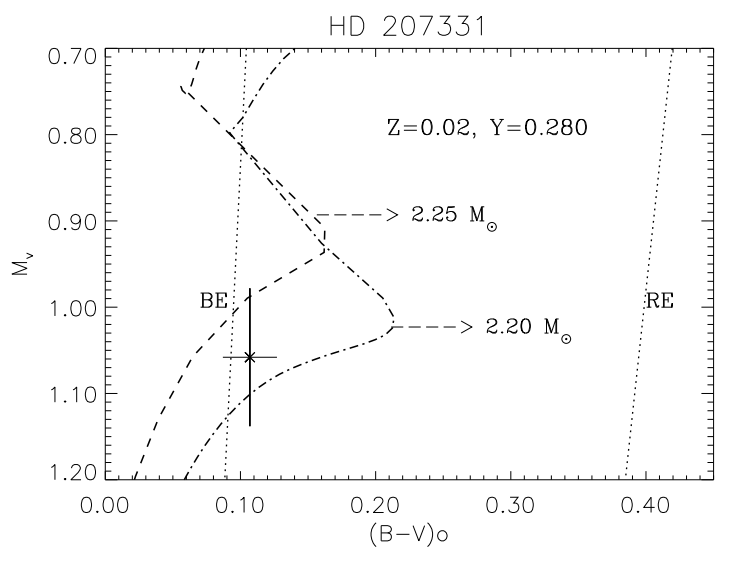

Figure 5: Position of HD 207331 in the HR diagram. Evolutive sequences of non-rotating models without overshooting are shown by dashed $\left(M / M_{\odot}=2.25\right)$ and dot-dashed lines $\left(M / M_{\odot}=2.20\right)$. The borders of the instability strip are shown by dotted lines.

We now use these models to derive possible radial and nonradial modes in order to obtain some insights into the pulsation behaviour of HD 207331. The adiabatic eigenfrequencies were computed using the code FILOU (Suárez \& Goupil, 2008). The following identifications of the modes are possible, but not unique: $f_{1}-(l=0, n=5), f_{2}-(l=2, n=2), f_{3}-$ $(l=1, n=4), f_{4}-(l=2, n=3)$. We note that since these identifications were computed from just few equilibrium models, they should be considered as preliminary. Moreover, we have neglected the rotational effects in spite the fact that HD 207331 is most likely a high rotating star. In fact, the rotation effects are important not only in the position of the star in a HR diagram (Pérez Hernández, et al., 1999) but also in the computation of oscillation frequencies (Suárez et al., 2009).

Nevertheless, these simple computations indicates that HD 207331 may pulsate in radial and non-radial modes typical among $\delta$ Scuti stars. Since the star is located near blue edge of instability strip, it could be pulsating in higher overtones than the fundamental.

\section{Conclusions}

A summary of the follow-up observations which led to the characterization of the new $\delta$ Scuti star HD 207331 has been presented. The star was observed from two observatories distributed in longitude around the Earth. An overall run of 53.8 $\mathrm{h}$ of useful data was collected from the two sites on nine observing nights. A period analysis reveals that HD 207331 is a multiperiodic pulsating star with at least four oscillation modes.

A simple comparison with theoretical modes has been performed. The star shows complicated pulsations as do most of the $\delta$ Scuti stars. HD 207331 seems to pulsate with low-order- $p$ modes typical among $\delta$ Scuti stars.

The analysis of a few low resolution spectra points to it being a fast rotating $\delta$ Scuti star of spectral type A0V. 
To date our observations represent the most extensive work on HD 207331.

\section{Acknowledgements}

This work has received financial support from the UNAM via PAPIIT grant IN114309. WJS acknowledges financial support from CONACyT by way of grant 49434-F. Based on observations collected at the $0.84 \mathrm{~m}$ telescope at the Observatorio Astronómico Nacional at San Pedro Mártir, Baja California, Mexico, and at the IAC- 80 telescope operated by the Instituto de Astrofísica de Canarias in the Spanish Observatorio del Teide. Special thanks are given to the technical staff and night assistants of the San Pedro Mártir and Teide Observatories. This research has made use of the SIMBAD database operated at the CDS, Strasbourg (France).

\section{References}

Breger, M., et al. 1993, A\&A, 271, 482

Bush, T., \& Hintz E.G. 2008, AJ, 136, 1061

Costa,J.E.S. et al. 2007, A\&A, 468, 637

Fox Machado, L., et al. 2002, A\&A, 382, 556

Fox Machado, L., et al. 2007, AJ, 134, 860

Fox Machado, L., et al. 2008, CoAst, 156, 27

Fox Machado, L., et al. 2006, A\&A, 446, 611

Fox Machado, L., et al. 2010, New Astronomy, 15, 397

Lenz, P., \& Breger, M. 2005, CoAst, 146, 53

Li, Z. P., et al. 2002, A\&A, 395, 873

Li, Z. P., et al. 2004, A\&A, 420, 283

Morel, P., 1997, A\&AS 124, 597

Pérez Hernández, F., et al. 1999, A\&A, 346, 586

Perryman, M. A. C., et al. 1997, A\&A 323, L49

Rodríguez, E., et al., 1994, A\&AS 106, 21

Schlegel, D.J., et al. 1998, ApJ, 500, 525

Schuster, W. J., Ochoa, J., Zurita, C., Fox Machado, L., 2008, IBVS No.5900, 8

Suárez, J.C., \& Goupil M.J., 2008, Ap\&SS,316, 155

Suárez, J.C., et al. 2009, ApJ, 690, 1401 